\title{
Third Wave Treatments for Functional Somatic Syndromes and Health Anxiety Across the Age Span: A Narrative Review
}

\author{
Lisbeth Frostholm ${ }^{\mathrm{a}}$, Charlotte Ulrikka Rask ${ }^{\mathrm{b}}$ \\ [a] Research Clinic for Functional Disorders and Psychosomatics, Aarhus University Hospital, Aarhus, Denmark. \\ [b] Child and Adolescent Psychiatry Department, Psychiatry, Aarhus University Hospital, Skejby, Denmark.
}

Clinical Psychology in Europe, 2019, Vol. 1(1), Article e32217, https://doi.org/10.32872/cpe.v1i1.32217

Received: 2018-12-06 • Accepted: 2019-02-13 • Published (VoR): 2019-03-29

Handling Editor: Winfried Rief, Division of Clinical Psychology and Psychotherapy, Department of Psychology, Philipps-University of Marburg, Marburg, Germany

Corresponding Author: Lisbeth Frostholm, Research Clinic for Functional Disorders and Psychosomatics, Aarhus University Hospital, Aarhus C, Denmark. E-mail: lisfro@rm.dk

\begin{abstract}
Background: Functional disorders (FD) are present across the age span and are commonly encountered in somatic health care. Psychological therapies have proven effective, but mostly the effects are slight to moderate. The advent of third wave cognitive behavioural therapies launched an opportunity to potentially improve treatments for FD.

Method: A narrative review of the literature on the application of mindfulness-based therapies (MBT) and Acceptance \& Commitment Therapy (ACT) in children and adult populations with FD.

Results: There were very few and mainly preliminary feasibility studies in children and adolescents. For adults there were relatively few trials of moderate to high methodological quality. Ten MBT randomised trials and 15 ACT randomised trials of which 8 were internet-delivered were identified for more detailed descriptive analysis. There was no evidence to suggest higher effects of third wave treatments as compared to CBT. For MBT, there seemed to be minor effects comparable to active control conditions. A few interventions combining second and third wave techniques found larger effects, but differences in outcomes, formats and dosage hamper comparability.

Conclusions: Third wave treatments are getting established in treatment delivery and may contribute to existing treatments for FD. Future developments could further integrate second and third wave treatments across the age span. Elements unambiguously targeting specific illness beliefs and exposure should be included. The benefit of actively engaging close relatives in the treatment not only among younger age groups but also in adults, as well as the effect of more multimodal treatment programmes including active rehabilitation, needs to be further explored.
\end{abstract}




\section{Keywords}

functional disorders, functional somatic syndromes, health anxiety, somatic symptom disorder, third wave treatments, mindfulness, acceptance and commitment therapy, narrative review

\section{Highlights}

- The methodological quality of third wave interventions for FD should be improved, especially in younger age groups.

- The effect of ACT interventions may be comparable to CBT in adults with FD.

- The evidence for third wave interventions in young people with FD is still very limited.

- Newer studies combining second and third wave treatments show some promise.

- Agreement on, and for child populations further development of, core outcomes, could help determine effect across studies.

Functional disorders (FD) can be defined as conditions where the individual's experiences of physical symptoms cause excessive discomfort and/or worry and where no adequate organ pathology in terms of conventional medical disease can be determined to explain the symptoms (Fink \& Rosendal, 2015). FD are a burden for sufferers and their families, they are difficult to treat and costly as they incur a high health expenditure and derived societal costs (Henningsen, Zipfel, Sattel, \& Creed, 2018).

\section{Diagnostic Classification}

Functional disorders can clinically be split into two overall categories (see Table 1).

The first category refers to conditions characterised by bodily distress, a now wellaccepted term to describe the phenomenon of clusters of disabling unspecific bodily symptoms often designated as functional somatic syndromes (FSS); the best known being chronic fatigue syndrome (CFS), fibromyalgia/chronic pain (FM/CP) and irritable bowel syndrome (IBS) (Fink \& Schröder, 2010). The second category refers to conditions dominated by health anxiety (HA), i.e. impairing illness worry and persisting ruminations about harbouring or getting serious illness (Fink et al., 2004). Although the two categories overlap in their clinical presentations and can be comorbid, the primary problem differs which has implications for the treatment focus.

In the psychiatric classifications ICD-10 (WHO, 1992) and DSM-IV (American Psychiatric Association, 1994), FD are mainly categorised under somatoform and related disorders. However, the terminology of these diagnoses has been criticised for being too exclusive in their diagnostic criteria as well as over-emphasising a mind-body dualism in contrast to the prevailing understanding of these disorders within an integrated biopsy- 
Table 1

Two Main Categories of Functional Disorders

\section{Disorders dominated by}

\begin{tabular}{lll}
\cline { 2 - 3 } Characteristics & \multicolumn{1}{c}{ Bodily distress (FSS) } & \multicolumn{1}{c}{ Health anxiety (HA) } \\
\hline Primary problem & $\begin{array}{l}\text { Experience of disabling physical } \\
\text { symptoms }\end{array}$ & $\begin{array}{l}\text { Experience of worries and anxiety } \\
\text { related to physical sensations }\end{array}$ \\
Functional impairment & $\begin{array}{l}\text { Severe physical disability } \\
\text { (e.g. sick leave, bedridden. In children } \\
\text { and adolescents often long-term school } \\
\text { absence) }\end{array}$ & $\begin{array}{l}\text { Less severe physical disability } \\
\text { (e.g. going to work serves as a distraction } \\
\text { from distressing thoughts. In children } \\
\text { and adolescents it will often be going to } \\
\text { school or playing computer games) }\end{array}$
\end{tabular}

Typical initial treatment Body can be fixed and the symptoms

Wish for $100 \%$ reassurance that they do expectations disappear not harbour a severe or deadly illness.

Note. FSS = Functional Somatic Syndromes; HA = Health Anxiety.

chosocial framework (Dimsdale, Sharma, \& Sharpe, 2011; Henningsen, Zipfel, \& Herzog, 2007). In the more recent DSM-5 (American Psychiatric Association, 2013), FD are classified primarily as somatic symptom disorders (SSD) with an added category of illness anxiety disorder designated to conditions with HA but without concurrent distressing bodily symptoms (in which case SSD is used). In contrast to ICD-10, developmental aspects are to some degree incorporated in DSM-5 as it specifies that in children, a single prominent symptom such as recurrent abdominal pain, headache, fatigue or nausea is more common than in adults. It also emphasises that parents' response to the symptoms is crucial as this may determine levels of associated distress and the extent to which medical help is sought.

In daily clinical practice, the psychiatric classifications are rarely used, as FD are primarily diagnosed in primary and specialised somatic health care. Thus, each medical specialty has developed its own classification leading to the use of a vast number of both unspecific symptom diagnoses as well as the previously mentioned FSS diagnoses. As a consequence, management in both the paediatric and adult health care settings is very heterogeneous, often formed by biomedical practices in each medical specialty and often not evidence-based. In addition, it is well-established that excessive biomedical treatment efforts cause iatrogenic harm in these conditions (Henningsen et al., 2007; Lindley, Glaser, \& Milla, 2005). 


\section{Developmental Aspects of FD}

Young children usually present a single prominent symptom (Domènech-Llaberia et al., 2004; Rask et al., 2009) such as abdominal pain, headaches, fatigue or muscle pains rather than the varied symptom presentation often seen in adults. The long-term prognosis varies from complete recovery to persistent symptoms into adulthood. With increasing age, full recovery seems to become more and more unlikely (Joyce, Hotopf, \& Wessely, 1997; Norris et al., 2017).

With respect to HA, key features such as symptom preoccupation and medical help seeking predominate mostly with the parents, although HA-like symptoms may present already in preschool children (Rask, Elberling, Skovgaard, Thomsen, \& Fink, 2012; Schulte \& Petermann, 2011). Also, preadolescents can report excessive illness worries with fears, beliefs and attitudes very similar to the cognitive and behavioural features of HA in adults (Eminson, Benjamin, Shortall, Woods, \& Faragher, 1996; Rask et al., 2016; van Geelen, Rydelius, \& Hagquist, 2015; Wright \& Asmundson, 2003). However, HA is still sparsely examined as a distinct concept in youth.

\section{Epidemiology}

Across the age span, the severity of both FSS and HA varies on a spectrum from mild and moderate to severely disabling conditions. New studies suggest that FSS affect $15 \%$ of the adult population, whereas approximately $2 \%$ of the population has very disabling conditions (Eliasen et al., 2018). In comparison, $4-10 \%$ of the general child and adolescent population experiences daily or high levels of impairing functional symptoms persisting for months or years (Hoftun, Romundstad, Zwart, \& Rygg, 2011; Janssens, Klis, Kingma, Oldehinkel, \& Rosmalen, 2014; Rask et al., 2009). The prevalence estimates for HA vary considerably across studies, but a recent study reported a prevalence of $3.4 \%$ (Sunderland, Newby, \& Andrews, 2013) in the general population. Around 8-9\% of the preadolescent general population reports high levels of illness worry (Rask et al., 2016), but prevalence estimates for HA as a disorder are not available in young age groups.

\section{Cognitive Behavioural Therapies for FD}

Chronicity, severity and multiplicity of symptoms are all predictors of poor prognosis (Rosendal et al., 2017). Therefore, timely and evidence-based treatment is essential for improving the long-term physical, psychosocial and financial consequences. Across age groups, patient-activating therapies are the most promising treatments, and cognitive behavioural therapy (CBT) has so far been the most prevailing in intervention studies (Abbott et al., 2018; Bonvanie et al., 2017; Henningsen et al., 2018). While moderate to large effect sizes (ES) have been reported for CBT-based treatment for HA (Hedman et al., 2011; Newby et al., 2018; Thomson \& Page, 2007; Weck, Neng, Schwind, \& Hofling, 
2015), improvements are only small to moderate for FSS in adults (Henningsen et al., 2018; van Dessel et al., 2014).

In children and adolescents, the use of CBT for HA has only been reported in a single case study (Roberts-Collins, 2016). With regard to FSS, existing studies have almost exclusively focused on CBT-based treatments for single symptoms or syndromes; primarily functional abdominal symptoms, chronic fatigue, tension-type headache, fibromyalgia or mixed pain complaints in children as young as 6 years of age (Abbott et al., 2018; Bonvanie et al., 2017). Overall, the ES are found to be somewhat larger than the corresponding estimates in adult studies (Bonvanie et al., 2017). This may indicate that children and adolescents are more susceptible to psychological treatments than adults or that young people present less chronic and/or severe FSS. However, the results should be interpreted with caution as the majority of these studies are quite small and heterogeneous with regard to e.g. inclusion criteria, setting, dose and type of delivered treatment and therapist experience (Abbott et al., 2018; Bonvanie et al., 2017).

Overall, these results, especially as to FSS, suggest that the efficacy of existing psychological treatments for FD could be improved. This has spurred interest in studies exploring the potential of the newer third wave behavioural therapies for these disorders.

\section{Treatment With Third Wave Psychological Therapies for FD}

\section{Mindfulness-Based Therapies (MBT)}

MBT translate meditation from Buddhism and other spiritual practices into clinical interventions. While classical CBT approaches tend to prioritise changing the content of private experiences like thoughts, MBT emphasise the awareness of thoughts, feelings and sensations as transient events that can potentially be problematic but do not have to be. Thus, compared to CBT, there is no explicit focus on behavioural activation or modification. In most interventions, mindfulness is taught in groups emphasising an experiential format with sharing of experiences in the enquiry phase after formal meditations. The most well-known MBT programmes are Mindfulness Based Stress Reduction (MBSR) and Mindfulness Based Cognitive Therapy (MBCT). The primary homework in most MBT is daily mindfulness practice.

MBT are proposed to work through at least four processes: 1) attention regulation, 2) body awareness, 3) emotion regulation and 4) change in self-perspective (Hölzel et al., 2011) (see Figure 1). 


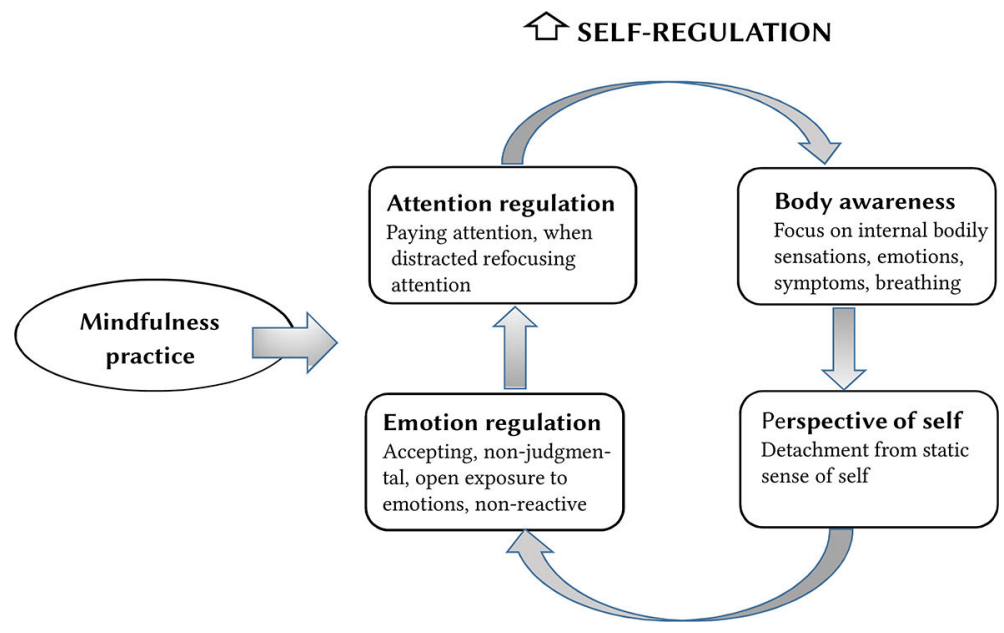

Figure 1. A model of proposed processes in mindfulness-based therapies.

Note. Adapted from Hölzel et al., 2011.

MBT could potentially change the perception of bodily symptoms through changes in interoception at a subconscious level and carry reductions in negative appraisal of symptoms. Furthermore, MBT might improve emotion regulation, which is proposed to play a prominent role in FSS (Dahlke, Sable, \& Andrasik, 2017) and as a by-product reduce comorbid anxiety and depression. In HA especially, one may hypothesise that mindfulness exercises can function as a direct exposure to anxiety-provoking bodily sensations and that the development of a more non-judgmental and accepting stance towards these bodily sensations may alleviate the symptom experience.

\section{Acceptance and Commitment Therapy}

The overarching goal of ACT is to increase psychological flexibility, defined as the ability to stay in contact with the present moment regardless of unpleasant thoughts, feelings and bodily sensations, while choosing one's behaviours based on the situation and personal values. In ACT, there are specific assumptions regarding the role of language for how human beings tend to handle 'the universal experience of pain' (loss, illness, conflict, and trauma) with avoidance of inner experience (Hayes, Luoma, Bond, Masuda, \& Lillis, 2006).

ACT proposes six core therapeutic processes which interact to promote psychological flexibility (see Figure 2). Experiential techniques such as mindfulness, defusion, metaphors and self-as-context exercises are used to illustrate and teach these processes. Compared to MBT, the kinship with second wave cognitive behavioural therapies is more obvious both in terms of format and content, e.g. the use of functional analyses, in which 
behaviours are analysed in terms of short- and long-term consequences (Hayes, 2016) and the focus on commitment to behaviour change.

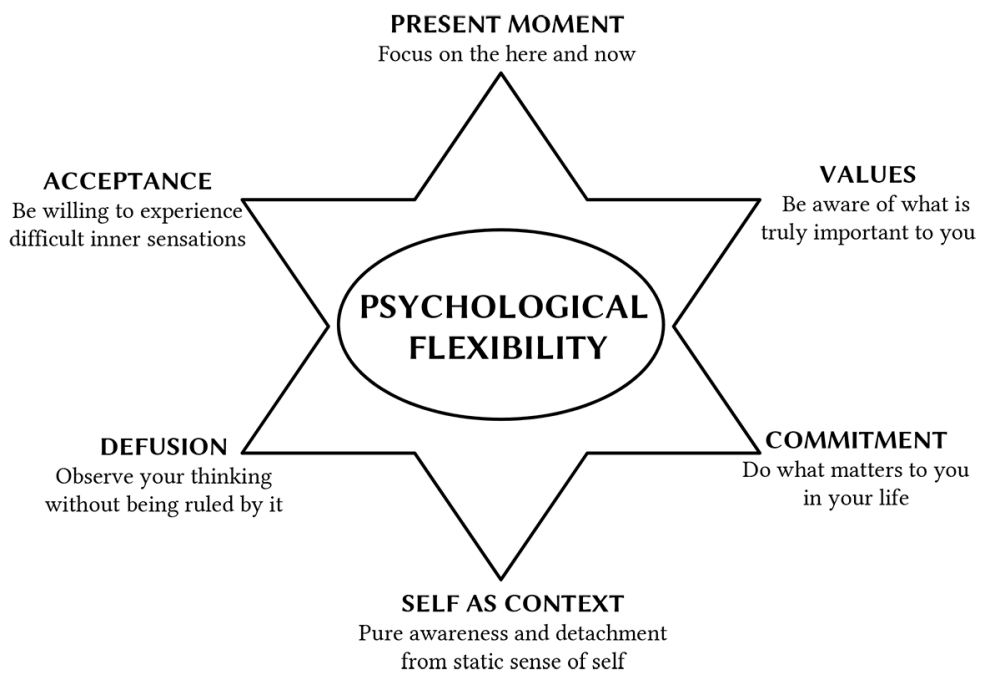

Figure 2. A model of the six core processes of ACT.

Note. Adapted from Hayes et al., 2006.

Specifically for FSS, a main treatment focus in ACT is on a behavioural shift from control and avoidance behaviours to choosing values-based actions even when aversive symptoms are present. Acceptance of bodily symptoms might both increase the engagement in behaviour change and lead to a reduction in symptom experience. In HA, where ruminations about bodily sensations are prominent (see Table 1), the focus on defusion from distressing illness-related thoughts could be helpful in alleviating the anxiety attached to illness labels such as cancer or sclerosis. Functional analysis may help foster a clearer understanding of the negative long-term effects of control and avoidance behaviours typical for HA (e.g. bodily checking and seeking information on symptoms on the internet).

\section{The Evidence-Base for MBT and ACT for HA and FSS}

An overview of the search methods and criteria for selection of studies for the current paper is provided in Table 2. 
Table 2

Search Methods and Criteria for Selection of Studies

- Publications on treatment outcome using Acceptance \& Commitment Therapy or mindfulness-based therapies for health anxiety and various functional somatic syndromes were identified in searches performed in September 2018 on PubMed by the help of a research librarian.

- The database was searched for English language studies using the terms 'Third wave' or 'Mindfulness-based stress reduction' or 'Mindfulness-based cognitive therapy' or 'MBCT' or 'MBSR' or 'Acceptance and Commitment Therapy' or 'Mindfulness' combined with 'Chronic Pain' or 'Fibromyalgia' or 'Fatigue Syndrome' or 'Irritable Bowel Syndrome' or 'Abdominal Pain' or 'Functional Gastrointestinal Disorders' or 'Somatoform Disorders' or 'Health anxiety' or 'Hypochondriasis' or 'Illness anxiety disorder' or 'Somatic symptom disorder'.

- For studies on adult populations, the search was restricted to systematic reviews and the reference lists of included studies were examined for additional eligible studies. The Web of Science was used for forward citation to identify additional papers. Only studies which randomised $\geq 50$ patients were included ${ }^{\mathrm{a}}$. With regard to chronic pain populations, studies were excluded if a substantial part of the population did not have an idiopathic or functional pain condition. Pure online self-help programmes with no therapist contact were not included.

- For child and adolescent papers the search terms were further combined with the terms 'child' or 'adolescent' or 'youth or 'paediatrics' or 'minor' or 'juvenile' or 'teen'. Based on the overall small number of studies no restriction was here applied with regard to study type.

- The methodological quality of the studies, including randomised controlled trials were rated using the psychotherapy outcome study rating scale (Öst, 2008).

${ }^{a}$ This cut-off was set in order to exclude studies which would better be classified as pilot trials (Bell, Whitehead, \& Julious, 2018).

\section{Evidence for HA in Adults and Children}

\section{MBT for HA}

The first preliminary results on the use of MBT in adults with HA were encouraging as a pilot study found significant improvements of MBCT on disease-related thoughts and somatic symptoms at 3-month follow-up (Lovas \& Barsky, 2010), and a qualitative study reported MBCT adapted to HA to be acceptable for the patients (McManus, Surawy, Muse, Vazquez-Montes, \& Williams, 2012; Williams, McManus, Muse, \& Williams, 2011). In the following RCT (McManus et al., 2012), 74 patients were randomised to either MBCT in addition to usual unrestricted service or usual unrestricted services alone (Table 3). 


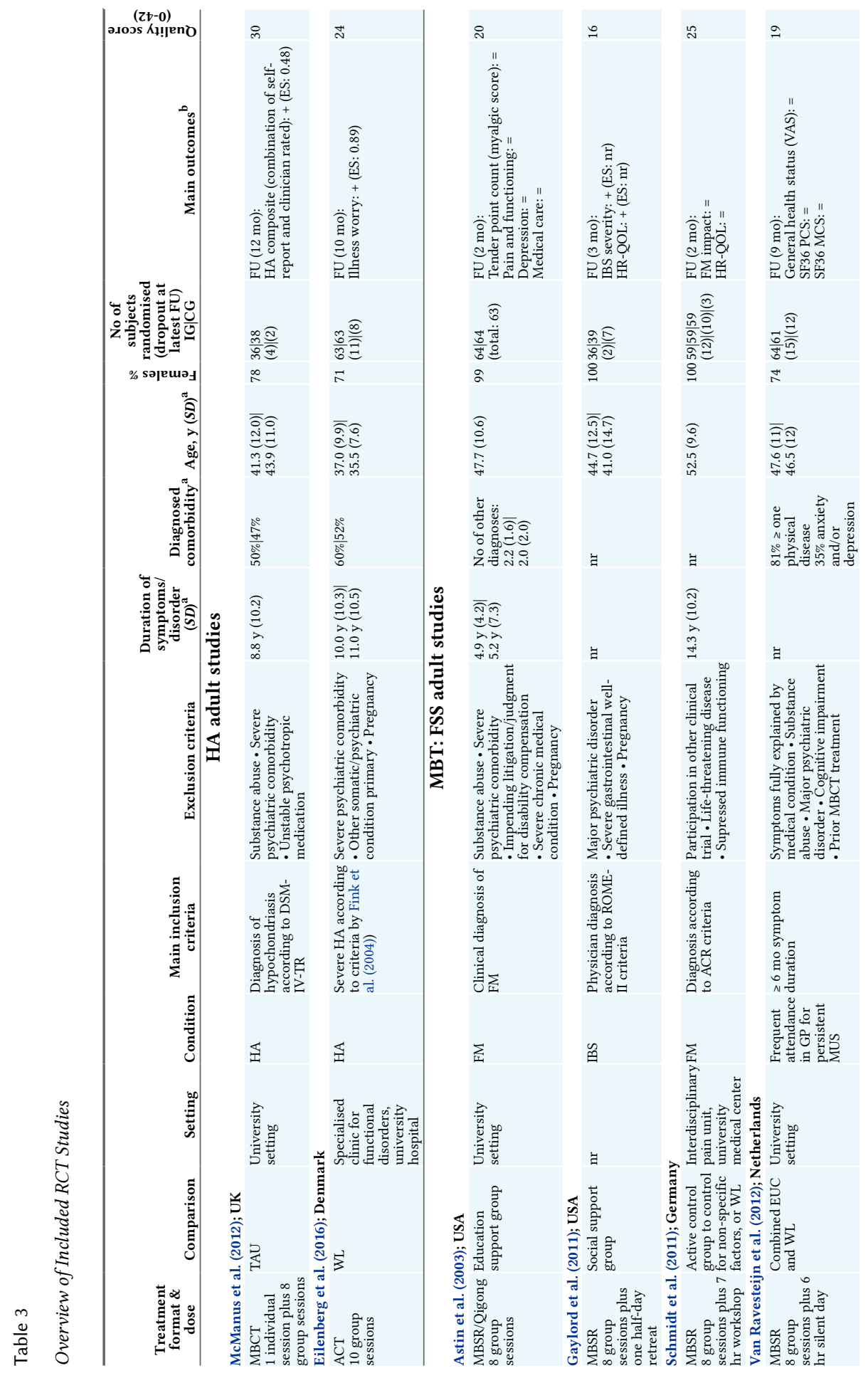




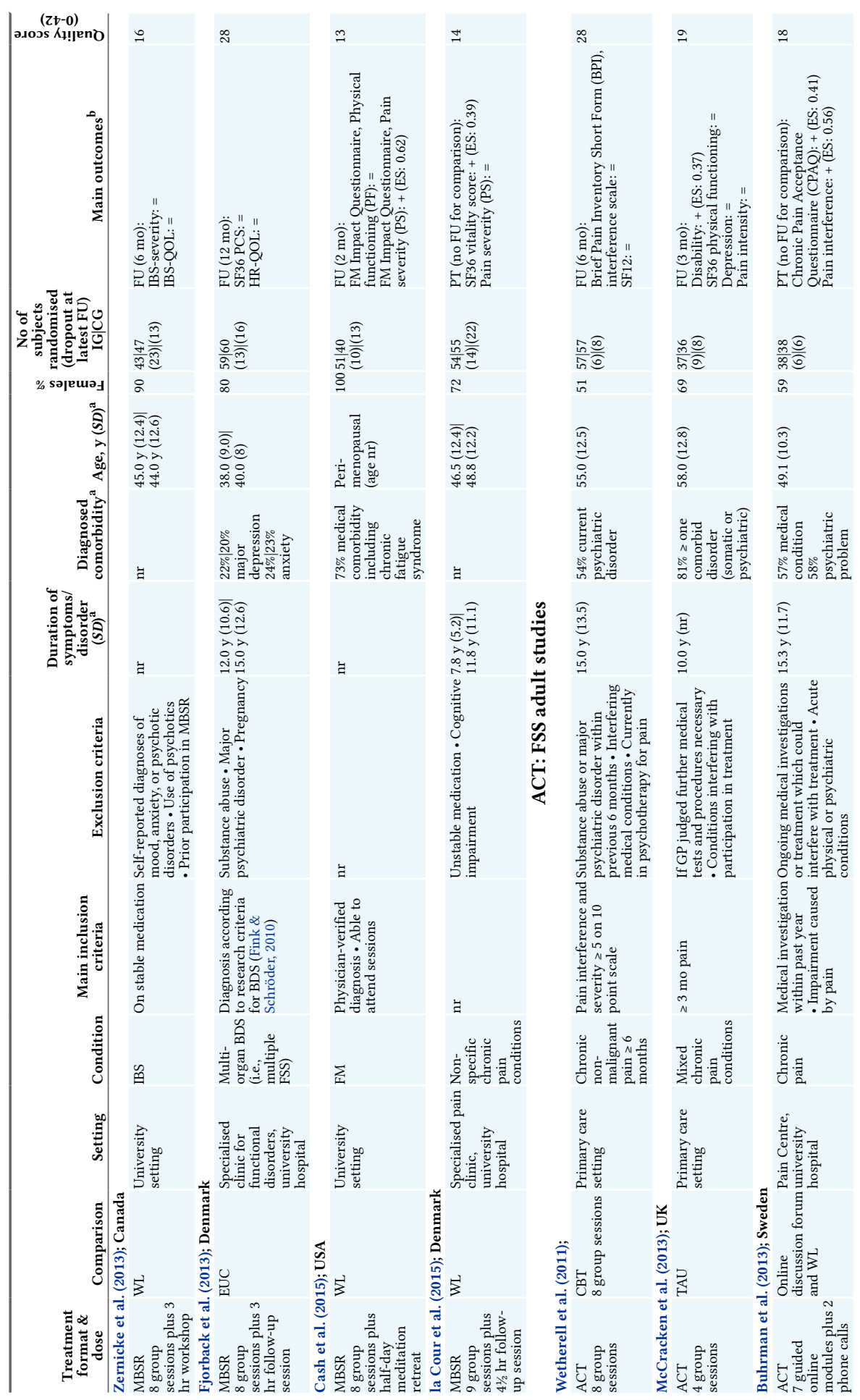




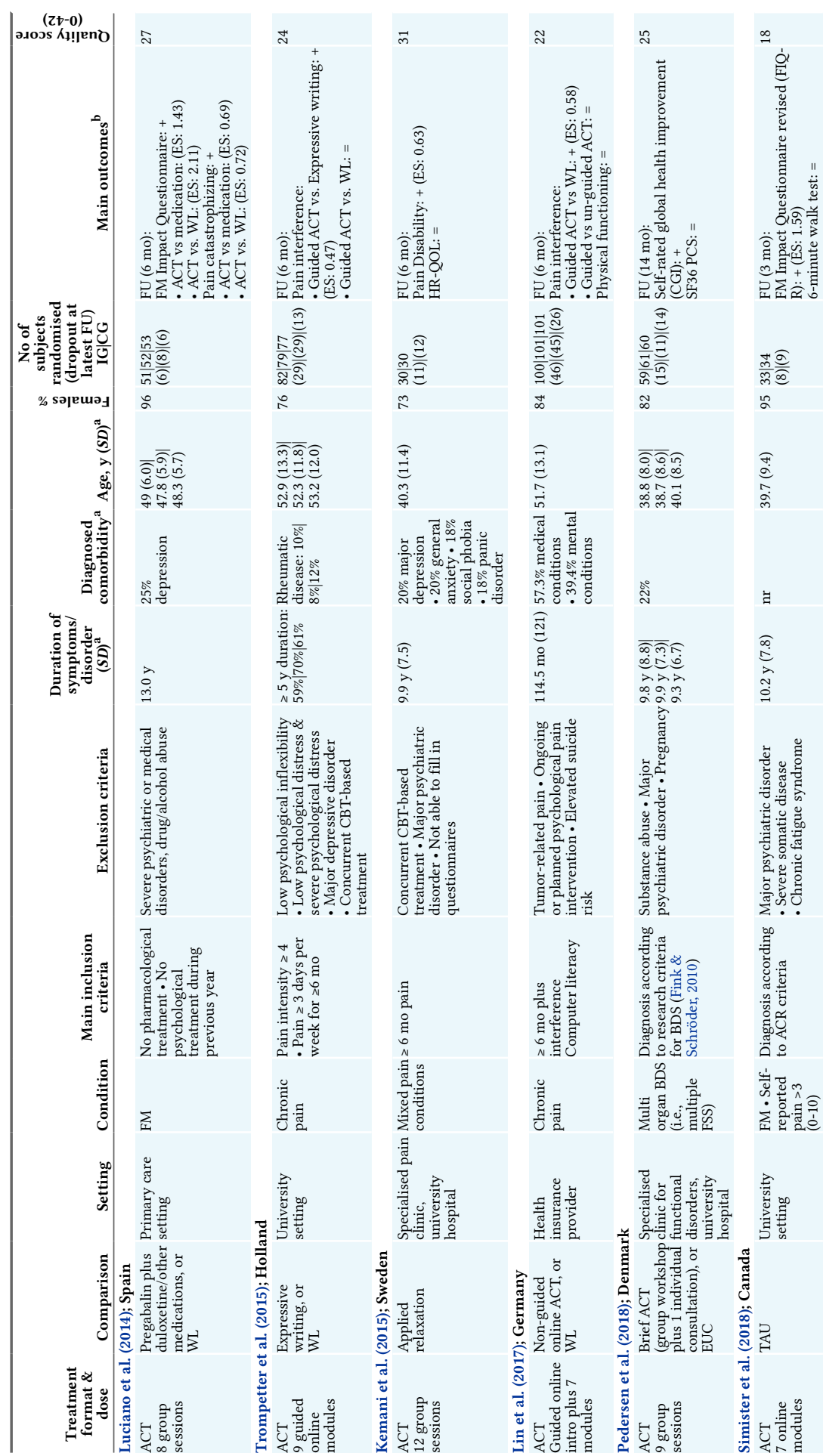




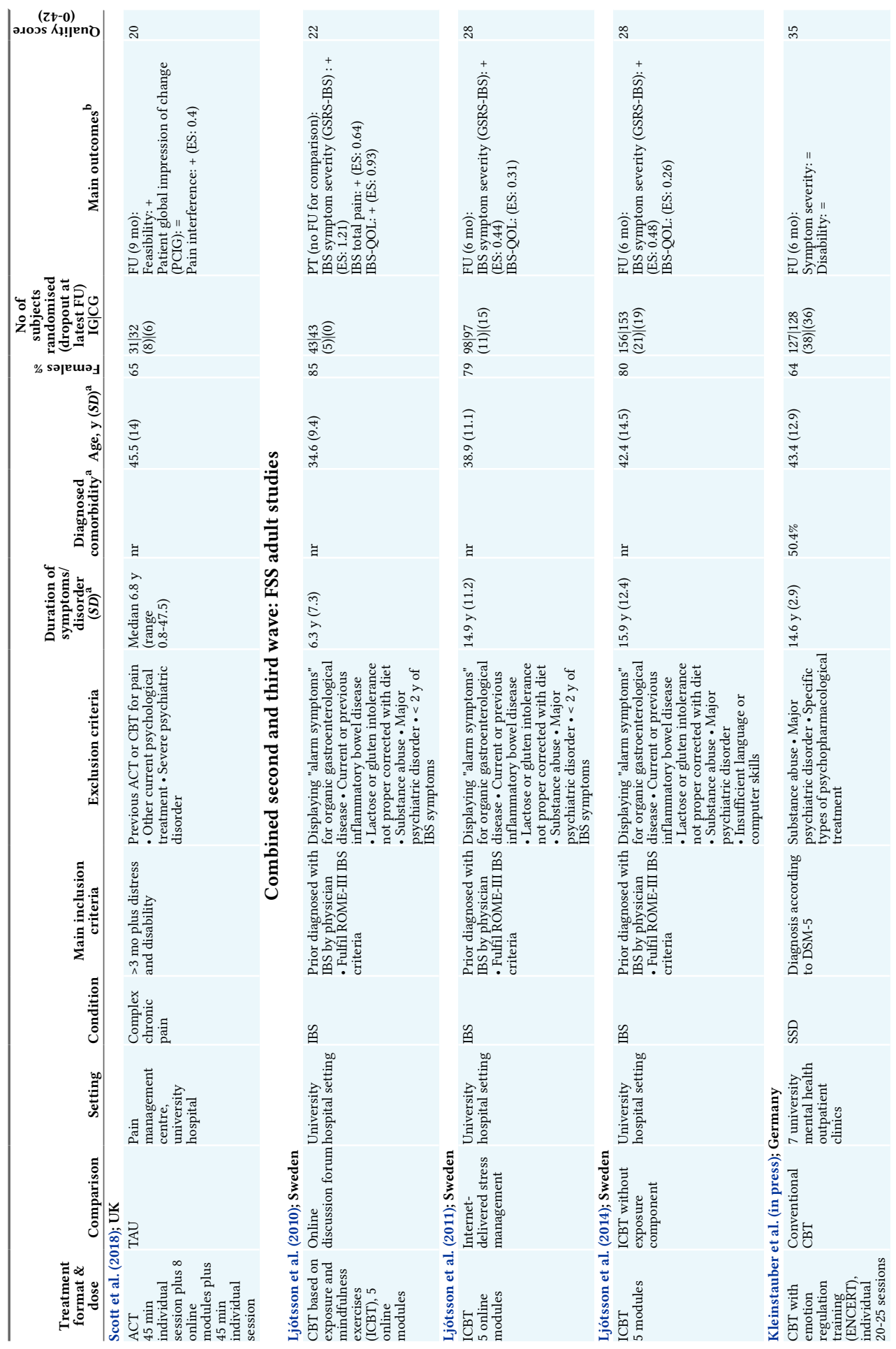




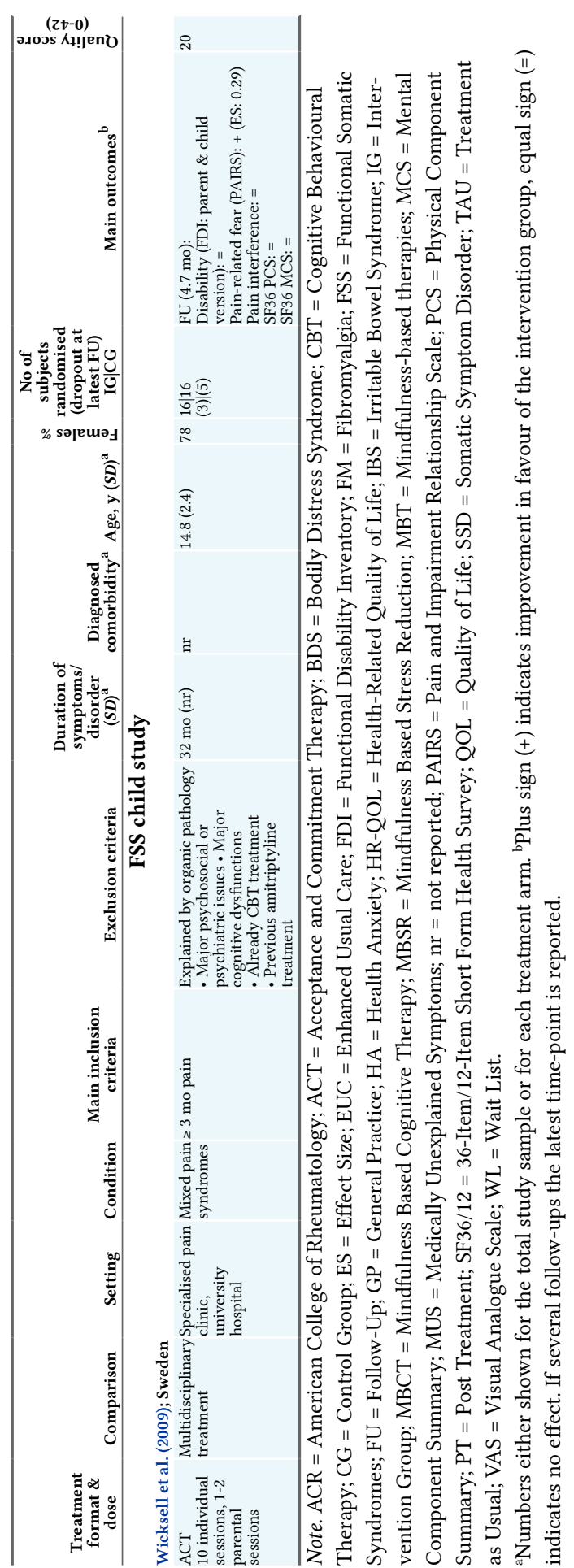


A medium ES of 0.48 was reported at one-year follow-up, which is at the lower end compared to existing CBT approaches. However, the drop-out rate was only 3\%, which is noticeably lower than rates reported in some of the CBT-based treatments for HA (e.g. 25\% from CBT in Greeven et al. (Greeven et al., 2007) and 35\% from CBT in Visser \& Bouman (Visser \& Bouman, 2001)).

\section{ACT for HA}

Only one RCT study using ACT for HA has been reported (Eilenberg, Fink, Jensen, Rief, \& Frostholm, 2016) (Table 3). The RCT was preceded by an uncontrolled pilot study suggesting that ACT group therapy may be an effective and acceptable treatment of HA (Eilenberg, Kronstrand, Fink, \& Frostholm, 2013). For the larger controlled study, the between-group effect sizes were large $(\mathrm{ES}=0.89$ ), and the treatment programme was well accepted by the patients. Thus, only 9 out of 135 eligible participants declined participation, and the drop-out rate in the ACT treatment was low as only $4(6 \%)$ out of 63 patients discontinued and one never attended the treatment. The programme was recently translated into an internet-based format, iACT for HA, with promising feasibility and efficacy reported in a pilot study (Hoffmann, Rask, Hedman-Lagerlof, Ljótsson, \& Frostholm, 2018). The results from a subsequent larger RCT with inclusion of 101 patients randomized to either iACT or an active control condition with an internet-delivered discussion forum are pending (Hoffmann, 2018). The literature search revealed no published treatment studies using any of the above approaches for children and adolescents with HA.

\section{Evidence for FSS in Adults}

\section{MBT for FSS}

Eight studies were located (Table 3). Three were on FM (Astin et al., 2003; Cash et al., 2015; Schmidt et al., 2011). One study focused on chronic pain (la Cour \& Petersen, 2015), 2 on IBS (Gaylord et al., 2011; Zernicke et al., 2013), 1 on persistent MUS (van Ravesteijn, Lucassen, Bor, van Weel, \& Speckens, 2013) and 1 on multi-organ BDS (Fjorback et al., 2013).

The smaller study on FM population found a potentially clinically relevant effect on symptom severity (Cash et al., 2015) of the MBSR program compared to treatment as usual (TAU). The two larger studies on FM (Astin et al., 2003; Schmidt et al., 2011) which both included an active control condition, an education support group and an education support including stretching and relaxation training, found no differences in their main outcomes (Table 3). Schmidt et al. thus concluded that MBSR cannot be recommended as a treatment for FM (Schmidt et al., 2011).

The study on chronic pain (la Cour \& Petersen, 2015) used an MBSR programme on top of usual care in a hospital-based pain clinic and found moderate effects on the main 
outcome of vitality, symptoms of anxiety and depression and control over pain immediately post-treatment but did not include long-term outcomes.

The two studies on IBS (Gaylord et al., 2011; Zernicke et al., 2013) both used MBSR and randomised 75 and 90 patients respectively. Both studies found clinically relevant within-group changes on the IBS symptom severity and other outcome measures. However, in the Zernicke study (Zernicke et al., 2013), which had a 6-month follow-up as opposed to 3 months in the Gaylord study (Gaylord et al., 2011), there was no significant difference between the MBSR and the waitlist at this final follow-up.

A Dutch study on high utilizers with persistent medically unexplained symptoms in primary care employed MBCT and found no effect on their primary outcome of general health status nine months after end of treatment. This also applied for the secondary outcomes except for the mindfulness skills of observing and describing (van Ravesteijn et al., 2013). The other study in the more severe spectrum (Fjorback et al., 2013) was also negative as there was no difference between the two groups even though the MBSR group had improved more on the main outcome of SF-36 Physical Component Summary towards the end of the active treatment period, whereas the enhanced treatment as usual caught up during the 1-year follow-up.

\section{ACT for FSS}

The majority of ACT studies in FSS have been conducted in chronic pain populations including FM, and the number of participants is surprisingly small. In the two most recent reviews on ACT for chronic pain, only five of 11 studies (Veehof, Trompetter, Bohlmeijer, \& Schreurs, 2016) and six of 10 studies (Hughes, Clark, Colclough, Dale, \& McMillan, 2017) respectively randomised at least 50 participants.

When including these larger trials, seven ACT studies were located for chronic pain, three of which were face-to-face (Kemani et al., 2015; McCracken, Sato, \& Taylor, 2013; Wetherell et al., 2011) and four of which were guided internet-delivered studies (Buhrman et al., 2013; Lin et al., 2017; Scott, Chilcot, Guildford, Daly-Eichenhardt, \& McCracken, 2018; Trompetter, Bohlmeijer, Veehof, \& Schreurs, 2015). Two studies were specifically on FM of which one was face-to-face (Luciano et al., 2014) and one guided internet-delivered (Simister et al., 2018). For multiple FSS, one study was located (Pedersen et al., 2018). That is, all in all 10 studies on FSS of which five were internetdelivered.

On top of the above distinct ACT interventions, one very recent study examined CBT with or without added acceptance-based emotion-regulation strategies for multiple medically unexplained symptoms (Kleinstauber et al., in press). Finally, three consecutive studies from one research group examined internet-delivered acceptance-based exposure therapy for IBS (Ljótsson et al., 2010; Ljótsson et al., 2011; Ljótsson et al., 2014).

Kemani and colleagues (Kemani et al., 2015) randomised 60 patients with chronic pain to either 12 90-minute weekly group sessions of ACT or applied relaxation (AR) but only 
obtained 6-month follow-up data on 37 participants. They found significantly larger effects of the ACT intervention immediately post-treatment on pain disability, but the AR group caught up in the follow-up period. A pilot RCT of a $4 \mathrm{x} 4$-hour primary care based ACT group intervention for chronic pain found only small effects compared to treatment as usual (McCracken et al., 2013). Wetherell (Wetherell et al., 2011) compared group CBT to the same amount of group ACT, all in all 12 hours, and overall found small and comparable effects of the two conditions on all outcomes (Wetherell et al., 2011). Interestingly, they found that participants assigned to CBT rated this as more credible after the first session, whereas ACT participants reported more satisfaction at the end of treatment.

Four studies examined the effect of guided internet-delivered ACT for chronic pain randomising 76, 238, 302, and 63 participants, respectively (Buhrman et al., 2013; Lin et al., 2017; Scott et al., 2018; Trompetter et al., 2015). The two largest trials were threearmed (Lin et al., 2017; Trompetter et al., 2015) (Table 3). Both of these studies found clinically relevant improvements of small to moderate effect of the ACT intervention compared to the control conditions, although the Trompetter study found unexpected improvements in the waitlist control (ibid). The results from these two larger internet-based studies were generally supported by the two smaller studies (Buhrman et al., 2013; Scott et al., 2018), even though the Buhrman study (Buhrman et al., 2013) included a large number of outcome measures given the small sample size.

The two studies on FM both found promising effects (Luciano et al., 2014; Simister et al., 2018). A group-based intervention carried out at primary health care centres in Spain was found superior on most outcome measures at 6-month follow-up compared to both recommended pharmacological treatment and to a waitlist control with large effects on fibromyalgia impact (Luciano et al., 2014). This finding was generally supported by the smaller study randomising 67 participants to either online ACT or treatment as usual (Simister et al., 2018).

Pedersen et al. (Pedersen et al., 2018) conducted a tree-armed intervention study examining group-based ACT with a brief ACT intervention (group workshop + individual session) and enhanced care (Pedersen et al., 2018) for patients with multiple FSS. They found effect of extended ACT on the primary outcome of patient-rated overall health improvement 14 months after randomisation but failed to replicate this finding on any of the secondary outcomes such as illness, worry, emotional distress and health-related quality of life.

A German multicentre study included patients with multiple medically unexplained symptoms (Kleinstauber et al., in press) and compared two active treatments, namely conventional CBT for FSS, which mainly focused on causing and maintaining factors and ENCERT: ENCERT was CBT with a primary focus on negative emotions as cause and consequence of FSS. This treatment arm included emotion regulation strategies such as acceptance and mindfulness-based strategies and cognitive reappraisal (ibid). They found medium to high effects on most outcomes in both conditions but also superior outcomes 
of ENCERT on a number of secondary outcomes such as health anxiety, symptom distress and emotion regulation skills.

Finally, a series of three studies on the same treatment programme for IBS (Ljótsson et al., 2010; Ljótsson et al., 2011; Ljótsson et al., 2014) combined acceptance strategies with mindfulness training and exposure. In the first modules of the treatment, they introduced mindfulness training and acceptance of symptoms together with a psychological model of IBS with the core message that behaviours which serve to avoid or control symptoms often increase the intensity of, and attention given to, symptoms (Ljótsson et al., 2010). The last phase of the treatment introduced exposure such as attending contexts where symptoms normally occur, exercises to provoke symptoms and abolishment of behaviours to control the occurrence of symptoms (ibid.). They found high effects of this treatment compared to an online discussion forum (Ljótsson et al., 2010). In a subsequent study, the treatment was found superior with medium effect sizes on several outcomes compared to stress management, which emphasised symptom control through relaxation, dietary changes and problem-solving skills (Ljótsson et al., 2011). Finally, in a disentanglement study, they examined the effect of the intervention with and without the final exposure phase of the treatment programme and found a medium effect size in favour of the inclusion of systematic exposure (Ljótsson et al., 2014).

\section{Evidence for FSS in Children and Adolescents}

\section{MBT for FSS}

Our search identified 8 studies on MBT for FSS in children; the first study published in 2013 (Jastrowski Mano et al., 2013). The studies were generally small ( $N$, range 6-21). Most used pilot designs and mainly examined a developmentally adapted version of the MBSR programme in tertiary care settings on children and adolescents in the age range from 12 to 18 years with mixed chronic pain conditions. Only one smaller study has been on young patients with various FSS including chronic fatigue (Ali et al., 2017).

Attrition and recruitment problems were described in five of the studies (Hesse, Holmes, Kennedy-Overfelt, Kerr, \& Giles, 2015; Jastrowski Mano et al., 2013; Lovas et al., 2017; Ruskin, Gagnon, Kohut, Stinson, \& Walker, 2017; Ruskin, Kohut, \& Stinson, 2015) as well as problems with obtaining sufficient post test data to draw valid conclusion about outcome (Ruskin, Gagnon, Kohut, Stinson, \& Walker, 2017). However, three other recent studies indicate better feasibility results with low attrition and high acceptability but heterogeneous results when it comes to potential efficacy (Ali et al., 2017; Chadi et al., 2016; Waelde et al., 2017). Ali et al. (Ali et al., 2017) conducted an open trial on 18 adolescents with various FSS and found preliminary evidence for the MBSR programme with regard to improvement of functional disability, symptom impact and anxiety with consistency between parent and child measures. Chadi et al. (Chadi et al., 2016) evaluated a combination of MBSR and MBCT on 20 female adolescents who were randomised to either an intervention group or a waitlist control group. They reported no improvements in psycho- 
logical or pain symptoms but did find significant reductions in pre and post-mindfulness session salivary cortisol levels. Waelde et al. (Waelde et al., 2017) conducted an open trial on 20 adolescents with chronic pain who received a six-week group intervention based on an adult programme named 'Inner Resources for Stress' combining meditation practices, breath-focused cue word repetition and visualisation. Functional disability and frequency of pain complaints improved with small effect sizes $(d=0.2-0.3)$. Though parents in the study did not receive any specific interventions, their worry about their child's pain decreased with a large effect size $(d=0.75)$.

\section{ACT for FSS}

Also with regard to ACT, the evidence is still sparse in younger age groups. We identified 6 ACT studies (Gauntlett-Gilbert, Connell, Clinch, \& McCracken, 2013; Kanstrup et al., 2016; Kemani, Kanstrup, Jordan, Caes, \& Gauntlett-Gilbert, 2018; Wicksell, Dahl, Magnusson, \& Olsson, 2005; Wicksell, Melin, Lekander, \& Olsson, 2009; Wicksell, Melin, \& Olsson, 2007) including only one smaller RCT (Wicksell et al., 2009) (Table 3). A seventh study included several modalities, i.e. CBT, ACT and multi-family therapy (Huestis et al., 2017). All studies relate primarily to adolescents diagnosed with various types of chronic idiopathic pain and four were performed at the same research centre.

Wicksell et al. were the first to describe an ACT-oriented outpatient intervention in young patients with high levels of pain-related disability; first in a case study (Wicksell et al., 2005), next in a case series on 14 adolescents (Wicksell et al., 2007) and subsequently in an RCT on 32 adolescents (mean age $14.8 \mathrm{yrs).} \mathrm{The} \mathrm{RCT} \mathrm{compared} 10$ sessions of ACT and one to two parent sessions with a multidisciplinary treatment including amitriptyline medication (Wicksell et al., 2009). Overall significant improvements with decreased disability were observed in all three studies, and specifically in the RCT, effects in favour of ACT were seen post-treatment in pain-related fear, pain interference and in quality of life. However, prolonged treatment in the control group complicated comparisons between the groups at follow-up assessments where all primary outcomes except pain-related fear became comparable (Table 3). The same research group later compared different formats of an extended version of this ACT programme, provided either individually ( $n=$ 18 ) or as group-based treatment $(n=12)$. Medium to large effects post-treatment were reported in both formats on pain interference, depression, pain reactivity and psychological flexibility as well as in parent pain reactivity and psychological flexibility post-treatment (Kanstrup et al., 2016).

In an uncontrolled trial (Gauntlett-Gilbert et al., 2013), 98 adolescents (mean age 15.6 yrs) with non-malignant pain underwent a 3-week residential multidisciplinary ACT treatment (approx. $90 \mathrm{hrs}$ ) in a specialised setting. The programme comprised physical conditioning, activity management and psychotherapy with promotion of acceptance of pain and related distress as well as engagement in values-consistent behaviour. Parent involvement was included in most sessions. The adolescents improved in self-reported 
functioning and objective physical performance at a 3-month follow-up. They were less anxious and catastrophic, attended school more regularly and used health care facilities less often. The programme was re-evaluated on another 164 patients as regards both adolescent and parental variables and the relationship between parental psychological flexibility and adolescent pain acceptance (Kemani et al., 2018). As in the former study, results indicated positive effects on the adolescents' functioning and pain acceptance but also a significant positive relationship between changes in parental psychological flexibility and adolescent pain acceptance.

A last study from 2017 describes the utility and outcomes of a multimodal intervention (CAPTIVES) including CBT, ACT and multi-family therapy in 17 youth (aged 13-17 years) with chronic pain and their parents (Huestis et al., 2017). The programme included weekly concurrent $60 \mathrm{~min}$. youth and parent groups, concluded with an additional 30 min. multi-family group session. The families found the programme engaging and constructive and large effects were reported on pain catastrophising, acceptance and protective parenting. Similar effects were found for functional disability, pain interference, fatigue, anxiety and depression.

Recently, a study protocol describing the design of a large RCT comparing groupbased ACT with enhanced usual care for adolescents with various FSS was published (Kallesøe et al., 2016). However, the results are still pending (personal communication).

\section{Discussion}

Even though third wave treatments are employed increasingly, there are still relatively few intervention studies in adults of moderate to high methodological quality in FD. Thus, in the updated 2016 review (Veehof et al., 2016) of a 2011 review (Veehof, Oskam, Schreurs, \& Bohlmeijer, 2011) on acceptance and mindfulness-based interventions, the authors concluded that the study quality had not improved in the five years since the first review, a finding supported by Öst's review on ACT for a broad range of conditions (Öst, 2014). As is the case with many emerging treatments, most studies in children and adolescents are small and uncontrolled in design.

\section{Evidence for Third Wave Treatment in HA}

For HA, the only two third wave RCTs on adults found a medium effect of MBCT tailored to HA (McManus et al., 2012) and high effect of ACT (Eilenberg et al., 2016). There were no studies in children or adolescents. Again, more studies are needed to replicate the findings from the above studies, especially the promising results of the ACT study, which reported high ES on the primary outcome and medium to high effect on most secondary outcomes and high retention of patients. It is worth noting that this study did, together with the vast majority of ACT interventions, include elements from second wave CBT 
such as psychoeducation using the vicious circle of anxiety and interoceptive exposure http://funktionellelidelser.dk/fileadmin/www.funktionellelidelser.au.dk/Publikationer/ ACT_Manual.pdf

With regard to younger age groups, HA is an emerging topic in the scientific literature. Integrating potential early childhood and family risk factors can help inform the development of specialised third wave therapies in children and adolescents (Thorgaard, Frostholm, \& Rask, 2018) as well as for parents with so-called health anxiety by proxy (Thorgaard et al., 2017), i.e. parents who present with excessive and seemingly unreasonable concern about their child's symptoms.

\section{Evidence for Third Wave Treatment in FSS}

Overall, there seems to be only minor effects of MBT in FSS. These findings are in line with the conclusions from a meta-analytic review that ES were higher for ACT therapies compared to MBT for the majority of the examined outcomes (Veehof et al., 2016). Some of the MBT studies in both adults and younger age groups are hampered by attrition, which may also suggest that MBT does not offer an alternative to second wave treatments in terms of retention. The two studies on MBT for IBS in adults (Gaylord et al., 2011; Zernicke et al., 2013) might suggest a bigger potential for this subgroup of patients given the clinically relevant change on the main outcome, but the effects may be transient. In children, there may be recruitment and retention problems for MBT programmes if the intervention is not properly modified and tailored according to developmental aspects. Children and adolescents in general require more explanation and rationale, shorter formal exercises (e.g. around 3-5 min compared to 20-45 min in adults) as well as a greater variety of practices if they are to engage fully (Perry-Parrish, Copeland-Linder, Webb, \& Sibinga, 2016; Thompson \& Gauntlett-Gilbert, 2008). From a clinical viewpoint, quite a few patients seem to benefit from MBT formats, and some of the target processes such as body awareness and emotional regulation could have promise. However, the mindfulness training may need to be embedded with other methods to prevent attrition and to increase effect.

There is no evidence to suggest that ACT is superior to CBT in FSS. More high quality studies are needed to conclude whether ACT is just as effective as CBT since the smaller studies, which have been included in many reviews, inherently have an increased risk of bias. There seems to be a potential in ACT-based therapist-guided internet-delivered interventions with a number of studies in chronic pain conditions reporting effects comparable to that of face-to-face interventions. Especially noteworthy here are the studies on acceptance-based exposure-based therapy for IBS (Ljótsson et al., 2010; Ljótsson et al., 2011), where acceptance-based techniques, mindfulness training and strict exposure training are combined to produce consistently large effects, and where the exposure element has been shown to add considerable effect (Ljótsson et al., 2014). Further studies 
could potentially benefit from tailoring symptom-specific exposure in the context of acceptance methods.

For conditions characterised by multiple symptoms from several organ systems, it was likewise the study which combined conventional CBT with third wave methods that had more convincing results (Kleinstauber et al., in press). Worth noting here is the dosage of treatment with 20-25 individual sessions as compared to e.g. 9 group sessions in the other trial on multiple symptoms (Pedersen et al., 2018). A secondary analysis of a group-based CBT intervention for multiple FSS (Schröder, Sharpe, \& Fink, 2015b) found higher effect in the subgroup of patients with fewest symptoms. This suggests that illness severity should be taken into account when designing interventions, and more extensive interventions may be needed in the severe spectrum of FSS.

With regard to children, the evidence is surprisingly low with small and mostly uncontrolled studies on paediatric chronic pain conditions. Therefore, it remains unclear whether observed effects reflect differences in samples, designs, instruments used, method of analysis or actual effects of different treatment modalities. However, the emphasis on experiential exercises and metaphors in ACT may render this approach particularly appropriate for children. Concepts that would normally be too abstract for children can become accessible through experience and metaphorical language (Coyne, McHugh, \& Martinez, 2011; Murrell, Coyne, \& Wilson, 2004). Still, larger and well-designed trials are needed to compare ACT to CBT interventions to examine the potential superiority of this approach in youth with FSS.

\section{Involvement of Family and Close Relatives in Third Wave Treatment}

The paediatric studies specifically emphasised inclusion of caregivers in treatment. This is supported by a number of studies reporting that parents may inadvertently reinforce maladaptive illness perceptions and illness behaviours in their child (Chow, Otis, \& Simons, 2016; Guite, McCue, Sherker, Sherry, \& Rose, 2011; Palermo, Valrie, \& Karlson, 2014; Simons, Smith, Kaczynski, \& Basch, 2015). Engaging parents may both help them ameliorate their own concerns and teach them how to reinforce and model adaptive behaviours. Recent studies have shown improvements in parental psychological flexibility of an 8-week ACT group programme (Wallace, Woodford, \& Connelly, 2016) and a onesession MBT workshop (Ruskin, Campbell, Stinson, \& Ahola Kohut, 2018) in parents of children with chronic pain, i.e. parents' abilities to accept their distress about their child's suffering and to focus on broader goals rather than being absorbed by worries about whether their child's pain improved.

In adult patients with FSS (CFS), their illness also seems to have a negative impact on the family (Higgins et al., 2015; Leonard \& Cano, 2006), and partners' responses may even influence the course of the disorder (Schmaling, Smith, \& Buchwald, 2000). Dynamic management involving family systems and close relatives to promote adaptive function- 
ing, quality of life and resilience may therefore also be an interesting focus for future studies on adults.

\section{Potential Challenges With Third Wave Treatment}

For FD, there is agreement that illness beliefs play a prominent role and that changes in beliefs such as perceived control (Christensen, Frostholm, Ornbol, \& Schröder, 2015) and fear-avoidance beliefs (Chalder, Goldsmith, White, Sharpe, \& Pickles, 2015) have been found to mediate the effect of CBT. One may speculate that there is a risk that the third wave meta-cognitive processes aimed at a general shift in perspective on inner experience and the self may not sufficiently address the specific cognitive beliefs that may perpetuate the symptoms for each individual patient. This risk may be further enhanced by the fact that all the included ACT studies, which were not internet-based, were groupbased (Kemani et al., 2015; Luciano et al., 2014; McCracken et al., 2013; Pedersen et al., 2018; Wetherell et al., 2011). Group-based therapy may have advantages in terms of providing support, promoting social skills and mirroring processes etc. but may also have risks in terms of not properly addressing the specific needs of each patient.

Large differences exist in use of outcome domains making it difficult to compare studies. Some ACT studies have used pain interference and pain acceptance as primary outcomes taking the point of departure that greater acceptance of symptoms and less interference of the pain in daily life are essential goals in ACT. Even though that may ring true from a theoretical perspective, we need more knowledge of the clinical importance of such changes. Other studies use syndrome-specific outcomes, hampering the comparability between syndromes. Including as a minimum the two numeric analog scale items on symptom intensity and symptom interference recommended by the European Network on Somatic Symptom Disorders in future adult trials (Rief et al., 2017) could have a major impact on the interpretation and comparability of studies. These scales encompass both the third wave focus on decreasing interference of symptoms as well as symptom reduction (see Figure 3).

For children and adolescent populations, the availability of validated questionnaires is extremely scant, and much more work is needed to develop and test such measures. 


\section{Symptom Intensity:}

During the last 7 days, the overall intensity of my bodily symptoms was:

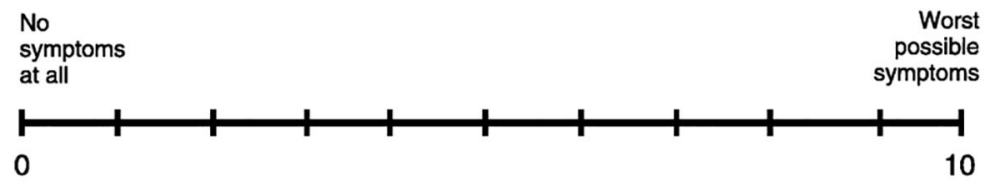

\section{Symptom Interference:}

During the last 7 days, my bodily symptoms interfered with daily life activities

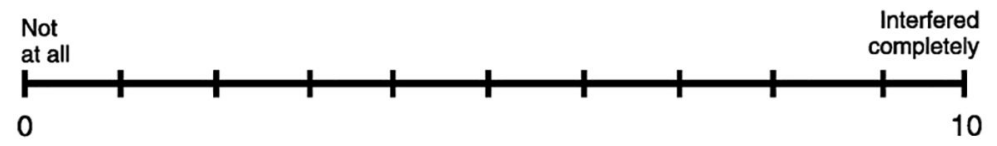

Figure 3. Two numeric rating scales recommended in future trials for FD.

Note. The scales are available at http://links.lww.com/PSYMED/A408 in more than 20 languages.

\section{Conclusion and Perspectives}

In sum, the evidence for third wave behavioural approaches for FD are still limited when it comes to larger controlled studies and very sparse and almost non-existing in younger age groups. There may have been hype surrounding the advent of third wave treatments which have hampered the ability among researchers and clinicians to communicate accurately about the advantages and disadvantages of these methods (Van Dam et al., 2018). Especially for children and adolescents, much progress remains to be made in empirically evaluating the effectiveness of third wave treatment. Thus, CBT-based programs still have much better evidence for this age group (Bonvanie et al., 2017).

There is often an unfortunate division between researchers and clinicians who study and treat adults with FD and those who work with children and adolescents with the same disorders. Joint efforts with mutual exchange of experiences and results could pave the way for further development of existing programmes such as the involvement of the family system in adult programmes.

Even though the field of FD will continue to be challenged by problems with diagnostic classification, agreement on joint outcomes across syndrome diagnoses and possibly more dismantling studies using e.g. single-case designs and/or experimental studies could also be a way forward to further explore which patient characteristics are compatible with certain approaches and techniques, both when it comes to children, adolescents and adults.

Finally, more studies explicitly combining methods from second and third wave approaches may be a promising avenue for patients across the age span. 
Funding: The authors have no funding to report.

Competing Interests: The authors have declared that no competing interests exist.

Acknowledgments: The authors have no support to report.

\section{References}

Abbott, R. A., Martin, A. E., Newlove-Delgado, T. V., Bethel, A., Whear, R. S., Thompson Coon, J., \& Logan, S. (2018). Recurrent abdominal pain in children: Summary evidence from 3 systematic reviews of treatment effectiveness. Fournal of Pediatric Gastroenterology and Nutrition, 67(1), 23-33. https://doi.org/10.1097/MPG.0000000000001922

Ali, A., Weiss, T. R., Dutton, A., McKee, D., Jones, K. D., Kashikar-Zuck, S., . . Shapiro, E. D. (2017). Mindfulness-based stress reduction for adolescents with functional somatic syndromes: A pilot cohort study. The fournal of Pediatrics, 183, 184-190. https://doi.org/10.1016/j.jpeds.2016.12.053

American Psychiatric Association. (1994). Diagnostic and statistical manual of mental disorders (4th ed.). Washington, DC, USA: Author.

American Psychiatric Association. (2013). Diagnostic and statistical manual of mental disorders (5th ed.). Arlington, VA, USA: Author.

Astin, J. A., Berman, B. M., Bausell, B., Lee, W. L., Hochberg, M., \& Forys, K. L. (2003). The efficacy of mindfulness meditation plus qigong movement therapy in the treatment of fibromyalgia: A randomized controlled trial. The fournal of Rheumatology, 30(10), 2257-2262.

Bell, M. L., Whitehead, A. L., \& Julious, S. A. (2018). Guidance for using pilot studies to inform the design of intervention trials with continuous outcomes. Clinical Epidemiology, 10, 153-157. https://doi.org/10.2147/CLEP.S146397

Bonvanie, I. J., Kallesøe, K. H., Janssens, K. A. M., Schröder, A., Rosmalen, J. G. M., \& Rask, C. U. (2017). Psychological interventions for children with functional somatic symptoms: A systematic review and meta-analysis. The fournal of Pediatrics, 187, 272-281.e17. https://doi.org/10.1016/j.jpeds.2017.03.017

Buhrman, M., Skoglund, A., Husell, J., Bergstrom, K., Gordh, T., Hursti, T., . . Andersson, G. (2013). Guided internet-delivered acceptance and commitment therapy for chronic pain patients: A randomized controlled trial. Behaviour Research and Therapy, 51(6), 307-315.

https://doi.org/10.1016/j.brat.2013.02.010

Cash, E., Salmon, P., Weissbecker, I., Rebholz, W. N., Bayley-Veloso, R., Zimmaro, L. A., . . Sephton, S. E. (2015). Mindfulness meditation alleviates fibromyalgia symptoms in women: Results of a randomized clinical trial. Annals of Behavioral Medicine, 49(3), 319-330. https://doi.org/10.1007/s12160-014-9665-0

Chadi, N., McMahon, A., Vadnais, M., Malboeuf-Hurtubise, C., Djemli, A., Dobkin, P. L., . . Haley, N. (2016). Mindfulness-based intervention for female adolescents with chronic pain: A pilot 
randomized trial. Fournal of the Canadian Academy of Child and Adolescent Psychiatry, 25(3), 159-168.

Chalder, T., Goldsmith, K. A., White, P. D., Sharpe, M., \& Pickles, A. R. (2015). Rehabilitative therapies for chronic fatigue syndrome: A secondary mediation analysis of the PACE trial. The Lancet: Psychiatry, 2(2), 141-152. https://doi.org/10.1016/S2215-0366(14)00069-8

Chow, E. T., Otis, J. D., \& Simons, L. E. (2016). The longitudinal impact of parent distress and behavior on functional outcomes among youth with chronic pain. The fournal of Pain, 17(6), 729-738. https://doi.org/10.1016/j.jpain.2016.02.014

Coyne, L. W., McHugh, L., \& Martinez, E. R. (2011). Acceptance and Commitment Therapy (ACT): Advances and applications with children, adolescents, and families. Child and Adolescent Psychiatric Clinics of North America, 20(2), 379-399. https://doi.org/10.1016/j.chc.2011.01.010

Christensen, S. S., Frostholm, L., Ornbol, E., \& Schröder, A. (2015). Changes in illness perceptions mediated the effect of cognitive behavioural therapy in severe functional somatic syndromes. Journal of Psychosomatic Research, 78(4), 363-370.

https://doi.org/10.1016/j.jpsychores.2014.12.005

Dahlke, L. A., Sable, J. J., \& Andrasik, F. (2017). Behavioral therapy: Emotion and pain, a common anatomical background. Neurological Sciences, 38(Suppl 1), 157-161. https://doi.org/10.1007/s10072-017-2928-3

Dimsdale, J., Sharma, N., \& Sharpe, M. (2011). What do physicians think of somatoform disorders? Psychosomatics, 52(2), 154-159. https://doi.org/10.1016/j.psym.2010.12.011

Domènech-Llaberia, E., Jane, C., Canals, J., Ballespi, S., Esparo, G., \& Garralda, E. (2004). Parental reports of somatic symptoms in preschool children: Prevalence and associations in a Spanish sample. Journal of the American Academy of Child and Adolescent Psychiatry, 43(5), 598-604. https://doi.org/10.1097/00004583-200405000-00013

Eilenberg, T., Fink, P., Jensen, J. S., Rief, W., \& Frostholm, L. (2016). Acceptance and commitment group therapy (ACT-G) for health anxiety: A randomized controlled trial. Psychological Medicine, 46(1), 103-115. https://doi.org/10.1017/S0033291715001579

Eilenberg, T., Kronstrand, L., Fink, P., \& Frostholm, L. (2013). Acceptance and commitment group therapy for health anxiety-Results from a pilot study. Fournal of Anxiety Disorders, 27(5), 461-468. https://doi.org/10.1016/j.janxdis.2013.06.001

Eliasen, M., Schröder, A., Fink, P., Kreiner, S., Dantoft, T. M., Poulsen, C. H., . . Jorgensen, T. (2018). A step towards a new delimitation of functional somatic syndromes: A latent class analysis of symptoms in a population-based cohort study. Fournal of Psychosomatic Research, 108, 102-117. https://doi.org/10.1016/j.jpsychores.2018.03.002

Eminson, M., Benjamin, S., Shortall, A., Woods, T., \& Faragher, B. (1996). Physical symptoms and illness attitudes in adolescents: An epidemiological study. Fournal of Child Psychology and Psychiatry, and Allied Disciplines, 37(5), 519-528.

https://doi.org/10.1111/j.1469-7610.1996.tb01438.x 
Fink, P., Ornbol, E., Toft, T., Sparle, K. C., Frostholm, L., \& Olesen, F. (2004). A new, empirically established hypochondriasis diagnosis. The American fournal of Psychiatry, 161(9), 1680-1691. https://doi.org/10.1176/appi.ajp.161.9.1680

Fink, P., \& Rosendal, M. (Eds.). (2015). Functional disorders and medically unexplained symptoms: Assessment and treatment. Aarhus, Denmark: Aarhus University Press.

Fink, P., \& Schröder, A. (2010). One single diagnosis, bodily distress syndrome, succeeded to capture 10 diagnostic categories of functional somatic syndromes and somatoform disorders. Journal of Psychosomatic Research, 68(5), 415-426. https://doi.org/10.1016/j.jpsychores.2010.02.004

Fjorback, L. O., Arendt, M., Ornbol, E., Walach, H., Rehfeld, E., Schröder, A., \& Fink, P. (2013). Mindfulness therapy for somatization disorder and functional somatic syndromes: Randomized trial with one-year follow-up. fournal of Psychosomatic Research, 74(1), 31-40. https://doi.org/10.1016/j.jpsychores.2012.09.006

Gauntlett-Gilbert, J., Connell, H., Clinch, J., \& McCracken, L. M. (2013). Acceptance and valuesbased treatment of adolescents with chronic pain: Outcomes and their relationship to acceptance. Fournal of Pediatric Psychology, 38(1), 72-81. https://doi.org/10.1093/jpepsy/jss098

Gaylord, S. A., Palsson, O. S., Garland, E. L., Faurot, K. R., Coble, R. S., Mann, J. D., . . Whitehead, W. E. (2011). Mindfulness training reduces the severity of irritable bowel syndrome in women: Results of a randomized controlled trial. The American fournal of Gastroenterology, 106(9), 1678-1688. https://doi.org/10.1038/ajg.2011.184

Greco, L. A., Blackledge, J. T., Coyne, L. W., \& Ehrenreich, J. (2005). Integrating acceptance and mindfulness into treatment for child and adolescent anxiety disorders: Acceptance and commitment therapy as an example. In S. M. Orsillo \& L. Roemer (Eds.), Acceptance and mindfulness-based approaches to anxiety: Conceptualization and treatment (pp. 301-322). Boston, MA, USA: Springer. https://doi.org/https://doi.org/10.1007/0-387-25989-9_12

Greeven, A., van Balkom, A. J., Visser, S., Merkelbach, J. W., van Rood, Y. R., van Dyck, R., . . . Spinhoven, P. (2007). Cognitive behavior therapy and paroxetine in the treatment of hypochondriasis: A randomized controlled trial. The American fournal of Psychiatry, 164(1), 91-99. https://doi.org/10.1176/ajp.2007.164.1.91

Guite, J. W., McCue, R. L., Sherker, J. L., Sherry, D. D., \& Rose, J. B. (2011). Relationships among pain, protective parental responses, and disability for adolescents with chronic musculoskeletal pain: The mediating role of pain catastrophizing. The Clinical fournal of Pain, 27(9), 775-781. https://doi.org/10.1097/AJP.0b013e31821d8fb4

Hayes, S. C. (2016). Acceptance and commitment therapy, relational frame theory, and the third wave of behavioral and cognitive therapies [Republished article]. Behavior Therapy, 47(6), 869-885. https://doi.org/10.1016/j.beth.2016.11.006

Hayes, S. C., Luoma, J. B., Bond, F. W., Masuda, A., \& Lillis, J. (2006). Acceptance and commitment therapy: Model, processes and outcomes. Behaviour Research and Therapy, 44(1), 1-25. https://doi.org/10.1016/j.brat.2005.06.006 
Hedman, E., Andersson, G., Andersson, E., Ljótsson, B., Ruck, C., Asmundson, G. J., \& Lindefors, N. (2011). Internet-based cognitive-behavioural therapy for severe health anxiety: Randomised controlled trial. The British fournal of Psychiatry, 198(3), 230-236.

https://doi.org/10.1192/bjp.bp.110.086843

Henningsen, P., Zipfel, S., \& Herzog, W. (2007). Management of functional somatic syndromes. Lancet, 369(9565), 946-955. https://doi.org/10.1016/S0140-6736(07)60159-7

Henningsen, P., Zipfel, S., Sattel, H., \& Creed, F. (2018). Management of functional somatic syndromes and bodily distress. Psychotherapy and Psychosomatics, 87(1), 12-31. https://doi.org/10.1159/000484413

Hesse, T., Holmes, L. G., Kennedy-Overfelt, V., Kerr, L. M., \& Giles, L. L. (2015). Mindfulness-based intervention for adolescents with recurrent headaches: A pilot feasibility study. Evidence-Based Complementary and Alternative Medicine, 2015, Article 508958. https://doi.org/10.1155/2015/508958

Higgins, K. S., Birnie, K. A., Chambers, C. T., Wilson, A. C., Caes, L., Clark, A. J., . . Campbell-Yeo, M. (2015). Offspring of parents with chronic pain: A systematic review and meta-analysis of pain, health, psychological, and family outcomes. Pain, 156(11), 2256-2266. https://doi.org/10.1097/j.pain.0000000000000293

Hoffmann, D. (2018). Internet-delivered acceptance and commitment therapy for health anxiety (Doctoral dissertation). Aarhus University, Aarhus, Denmark.

Hoffmann, D., Rask, C. U., Hedman-Lagerlof, E., Ljótsson, B., \& Frostholm, L. (2018). Development and feasibility testing of internet-delivered acceptance and commitment therapy for severe health anxiety: Pilot study. FMIR Mental Health, 5(2), Article e28. https://doi.org/10.2196/mental.9198

Hoftun, G. B., Romundstad, P. R., Zwart, J. A., \& Rygg, M. (2011). Chronic idiopathic pain in adolescence-High prevalence and disability: The young HUNT study 2008. Pain, 152(10), 2259-2266. https://doi.org/10.1016/j.pain.2011.05.007

Hölzel, B. K., Lazar, S. W., Gard, T., Schuman-Olivier, Z., Vago, D. R., \& Ott, U. (2011). How does mindfulness meditation work? Proposing mechanisms of action from a conceptual and neural perspective. Perspectives on Psychological Science, 6(6), 537-559.

https://doi.org/10.1177/1745691611419671

Huestis, S. E., Kao, G., Dunn, A., Hilliard, A. T., Yoon, I. A., Golianu, B., \& Bhandari, R. P. (2017). Multi-family pediatric pain group therapy: Capturing acceptance and cultivating change. Children, 4(12), Article 106. https://doi.org/10.3390/children4120106

Hughes, L. S., Clark, J., Colclough, J. A., Dale, E., \& McMillan, D. (2017). Acceptance and commitment therapy (ACT) for chronic pain: A systematic review and meta-analyses. The Clinical fournal of Pain, 33(6), 552-568. https://doi.org/10.1097/AJP.00000000000000425

Janssens, K. A., Klis, S., Kingma, E. M., Oldehinkel, A. J., \& Rosmalen, J. G. (2014). Predictors for persistence of functional somatic symptoms in adolescents. The fournal of Pediatrics, 164(4), 900-905.e2. https://doi.org/10.1016/j.jpeds.2013.12.003 
Jastrowski Mano, K. E., Salamon, K. S., Hainsworth, K. R., Anderson Khan, K. J., Ladwig, R. J., Davies, W. H., \& Weisman, S. J. (2013). A randomized, controlled pilot study of mindfulnessbased stress reduction for pediatric chronic pain. Alternative Therapies in Health and Medicine, 19(6), 8-14.

Joyce, J., Hotopf, M., \& Wessely, S. (1997). The prognosis of chronic fatigue and chronic fatigue syndrome: A systematic review. QfM, 90(3), 223-233. https://doi.org/10.1093/qjmed/90.3.223

Kallesøe, K. H., Schröder, A., Wicksell, R. K., Fink, P., Ornbol, E., \& Rask, C. U. (2016). Comparing group-based acceptance and commitment therapy (ACT) with enhanced usual care for adolescents with functional somatic syndromes: A study protocol for a randomised trial. BMF Open, 6(9), Article e012743. https://doi.org/10.1136/bmjopen-2016-012743

Kanstrup, M., Wicksell, R. K., Kemani, M., Wiwe Lipsker, C., Lekander, M., \& Holmstrom, L. (2016). A clinical pilot study of individual and group treatment for adolescents with chronic pain and their parents: Effects of acceptance and commitment therapy on functioning. Children, 3(4), Article 30. https://doi.org/10.3390/children3040030

Kemani, M. K., Kanstrup, M., Jordan, A., Caes, L., \& Gauntlett-Gilbert, J. (2018). Evaluation of an intensive interdisciplinary pain treatment based on acceptance and commitment therapy for adolescents with chronic pain and their parents: A nonrandomized clinical trial. fournal of Pediatric Psychology, 43(9), 981-994. https://doi.org/10.1093/jpepsy/jsy031

Kemani, M. K., Olsson, G. L., Lekander, M., Hesser, H., Andersson, E., \& Wicksell, R. K. (2015). Efficacy and cost-effectiveness of acceptance and commitment therapy and applied relaxation for longstanding pain: A randomized controlled trial. The Clinical fournal of Pain, 31(11), 1004-1016. https://doi.org/10.1097/AJP.0000000000000203

Kleinstauber, M., Bailer, J., Brünahl, C., Berking, M., Erkic, M., Gitzen, H., . . Weiss, F., Rief, W. (in press). Cognitive behavior therapy enriched with emotion regulation training (ENCERT) versus cognitive behavior therapy only for patients with multiple medically unexplained symptoms: A multi-center, randomized, phase 3 trial.

la Cour, P., \& Petersen, M. (2015). Effects of mindfulness meditation on chronic pain: A randomized controlled trial. Pain Medicine, 16(4), 641-652. https://doi.org/10.1111/pme.12605

Leonard, M. T., \& Cano, A. (2006). Pain affects spouses too: Personal experience with pain and catastrophizing as correlates of spouse distress. Pain, 126(1-3), 139-146. https://doi.org/10.1016/j.pain.2006.06.022

Lin, J., Paganini, S., Sander, L., Luking, M., Ebert, D. D., Buhrman, M., . . Baumeister, H. (2017). An internet-based intervention for chronic pain. Deutsches Ärzteblatt International, 114(41), 681-688. https://doi.org/10.3238/arztebl.2017.0681

Lindley, K. J., Glaser, D., \& Milla, P. J. (2005). Consumerism in healthcare can be detrimental to child health: Lessons from children with functional abdominal pain. Archives of Disease in Childhood, 90(4), 335-337. https://doi.org/10.1136/adc.2003.032524

Ljótsson, B., Falk, L., Vesterlund, A. W., Hedman, E., Lindfors, P., Ruck, C., . . Andersson, G. (2010). Internet-delivered exposure and mindfulness based therapy for irritable bowel syndrome-A 
randomized controlled trial. Behaviour Research and Therapy, 48(6), 531-539.

https://doi.org/10.1016/j.brat.2010.03.003

Ljótsson, B., Hedman, E., Andersson, E., Hesser, H., Lindfors, P., Hursti, T., . . Andersson, G. (2011).

Internet-delivered exposure-based treatment vs. stress management for irritable bowel syndrome: A randomized trial. The American fournal of Gastroenterology, 106(8), 1481-1491.

https://doi.org/10.1038/ajg.2011.139

Ljótsson, B., Hesser, H., Andersson, E., Lackner, J. M., El Alaoui, S., Falk, L., . . Hedman, E. (2014).

Provoking symptoms to relieve symptoms: A randomized controlled dismantling study of exposure therapy in irritable bowel syndrome. Behaviour Research and Therapy, 55, 27-39. https://doi.org/10.1016/j.brat.2014.01.007

Lovas, D. A., \& Barsky, A. J. (2010). Mindfulness-based cognitive therapy for hypochondriasis, or severe health anxiety: A pilot study. Fournal of Anxiety Disorders, 24(8), 931-935. https://doi.org/10.1016/j.janxdis.2010.06.019

Lovas, D. A., Pajer, K., Chorney, J. M., Vo, D. X., Howlett, M., Doyle, A., \& Huber, A. (2017). Mindfulness for adolescent chronic pain: A pilot feasibility study. Fournal of Child and Adolescent Mental Health, 29(2), 129-136. https://doi.org/10.2989/17280583.2017.1355807

Luciano, J. V., Guallar, J. A., Aguado, J., Lopez-Del-Hoyo, Y., Olivan, B., Magallon, R., . . GarciaCampayo, J. (2014). Effectiveness of group acceptance and commitment therapy for fibromyalgia: A 6-month randomized controlled trial (EFFIGACT study). Pain, 155(4), 693-702. https://doi.org/10.1016/j.pain.2013.12.029

McCracken, L. M., Sato, A., \& Taylor, G. J. (2013). A trial of a brief group-based form of acceptance and commitment therapy (ACT) for chronic pain in general practice: Pilot outcome and process results. The fournal of Pain, 14(11), 1398-1406. https://doi.org/10.1016/j.jpain.2013.06.011

McManus, F., Surawy, C., Muse, K., Vazquez-Montes, M., \& Williams, J. M. (2012). A randomized clinical trial of mindfulness-based cognitive therapy versus unrestricted services for health anxiety (hypochondriasis). Journal of Consulting and Clinical Psychology, 80(5), 817-828. https://doi.org/10.1037/a0028782

Murrell, A. R., Coyne, L. W., \& Wilson, K. G. (2004). ACT with children, adolescents and their parents. In S. C. Hayes \& K. D. Strosahl (Eds.), A practical guide to acceptance and commitment therapy (pp. 249-274). New York, NY, USA: Springer. https://doi.org/https://doi.org/10.1007/978-0-387-23369-7_10

Newby, J. M., Smith, J., Uppal, S., Mason, E., Mahoney, A. E. J., \& Andrews, G. (2018). Internet-based cognitive behavioral therapy versus psychoeducation control for illness anxiety disorder and somatic symptom disorder: A randomized controlled trial. fournal of Consulting and Clinical Psychology, 86(1), 89-98. https://doi.org/10.1037/ccp0000248

Norris, T., Collin, S. M., Tilling, K., Nuevo, R., Stansfeld, S. A., Sterne, J. A., . . Crawley, E. (2017). Natural course of chronic fatigue syndrome/myalgic encephalomyelitis in adolescents. Archives of Disease in Childhood, 102(6), 522-528. https://doi.org/10.1136/archdischild-2016-311198 
Öst, L. G. (2008). Efficacy of the third wave of behavioral therapies: A systematic review and metaanalysis. Behaviour Research and Therapy, 46(3), 296-321.

https://doi.org/10.1016/j.brat.2007.12.005

Öst, L. G. (2014). The efficacy of acceptance and commitment therapy: An updated systematic review and meta-analysis. Behaviour Research and Therapy, 61, 105-121.

https://doi.org/10.1016/j.brat.2014.07.018

Palermo, T. M., Valrie, C. R., \& Karlson, C. W. (2014). Family and parent influences on pediatric chronic pain: A developmental perspective. The American Psychologist, 69(2), 142-152. https://doi.org/10.1037/a0035216

Pedersen, H. F., Agger, J. L., Frostholm, L., Jensen, J. S., Ornbol, E., Fink, P., \& Schröder, A. (2018). Acceptance and commitment group therapy for patients with multiple functional somatic syndromes: A three-armed trial comparing ACT in a brief and extended version with enhanced care - CORRIGENDUM. Psychological Medicine, 48(16), 2804. https://doi.org/10.1017/S0033291718002799

Perry-Parrish, C., Copeland-Linder, N., Webb, L., \& Sibinga, E. M. (2016). Mindfulness-based approaches for children and youth. Current Problems in Pediatric and Adolescent Health Care, 46(6), 172-178. https://doi.org/10.1016/j.cppeds.2015.12.006

Rask, C. U., Elberling, H., Skovgaard, A. M., Thomsen, P. H., \& Fink, P. (2012). Parental-reported health anxiety symptoms in 5- to 7-year-old children: The Copenhagen Child Cohort CCC 2000. Psychosomatics, 53(1), 58-67. https://doi.org/10.1016/j.psym.2011.05.006

Rask, C. U., Munkholm, A., Clemmensen, L., Rimvall, M. K., Ornbol, E., Jeppesen, P., \& Skovgaard, A. M. (2016). Health anxiety in preadolescence-Associated health problems, healthcare expenditure, and continuity in childhood. Journal of Abnormal Child Psychology, 44(4), 823-832. https://doi.org/10.1007/s10802-015-0071-2

Rask, C. U., Olsen, E. M., Elberling, H., Christensen, M. F., Ornbol, E., Fink, P., . . Skovgaard, A. M. (2009). Functional somatic symptoms and associated impairment in 5-7-year-old children: The Copenhagen Child Cohort 2000. European fournal of Epidemiology, 24(10), 625-634. https://doi.org/10.1007/s10654-009-9366-3

Rief, W., Burton, C., Frostholm, L., Henningsen, P., Kleinstauber, M., Kop, W., . . van der FeltzCornelis, C. (2017). Core outcome domains for clinical trials on somatic symptom disorder, bodily distress disorder, and functional somatic syndromes: European network on somatic symptom disorders recommendations. Psychosomatic Medicine, 79(9), 1008-1015. https://doi.org/10.1097/PSY.0000000000000502

Roberts-Collins, C. (2016). A case study of an adolescent with health anxiety and OCD, treated using CBT: Single-case experimental design. fournal of Child and Adolescent Psychiatric Nursing, 29(2), 95-104. https://doi.org/10.1111/jcap.12136

Rosendal, M., Olde Hartman, T. C., Aamland, A., van der Horst, H., Lucassen, P., Budtz-Lilly, A., \& Burton, C. (2017). "Medically unexplained" symptoms and symptom disorders in primary care: Prognosis-based recognition and classification. BMC Family Practice, 18(1), Article 18. https://doi.org/10.1186/s12875-017-0592-6 
Ruskin, D., Campbell, L., Stinson, J., \& Ahola Kohut, S. (2018). Changes in parent psychological flexibility after a one-time mindfulness-based intervention for parents of adolescents with persistent pain conditions. Children, 5(9), Article 121. https://doi.org/10.3390/children5090121

Ruskin, D. A., Gagnon, M. M., Kohut, S. A., Stinson, J. N., \& Walker, K. S. (2017). A mindfulness program adapted for adolescents with chronic pain: Feasibility, acceptability, and initial outcomes. The Clinical fournal of Pain, 33(11), 1019-1029.

https://doi.org/10.1097/AJP.0000000000000490

Ruskin, D., Kohut, A. S., \& Stinson, J. (2015). The development of a mindfulness-based stress reduction group for adolescents with chronic pain. Journal of Pain Management, 7(4), 301-312.

Schmaling, K. B., Smith, W. R., \& Buchwald, D. S. (2000). Significant other responses are associated with fatigue and functional status among patients with chronic fatigue syndrome.

Psychosomatic Medicine, 62(3), 444-450. https://doi.org/10.1097/00006842-200005000-00018

Schmidt, S., Grossman, P., Schwarzer, B., Jena, S., Naumann, J., \& Walach, H. (2011). Treating fibromyalgia with mindfulness-based stress reduction: Results from a 3-armed randomized controlled trial. Pain, 152(2), 361-369. https://doi.org/10.1016/j.pain.2010.10.043

Schröder, A., Sharpe, M., \& Fink, P. (2015). Medically unexplained symptom management. Lancet, 2(7), 587-588. https://doi.org/10.1016/S2215-0366(15)00233-3

Schulte, I. E., \& Petermann, F. (2011). Somatoform disorders: 30 years of debate about criteria! What about children and adolescents? fournal of Psychosomatic Research, 70(3), 218-228.

https://doi.org/10.1016/j.jpsychores.2010.08.005

Scott, W., Chilcot, J., Guildford, B., Daly-Eichenhardt, A., \& McCracken, L. M. (2018). Feasibility randomized-controlled trial of online acceptance and commitment therapy for patients with complex chronic pain in the United Kingdom. European fournal of Pain, 22(8), 1473-1484. https://doi.org/10.1002/ejp.1236

Simister, H. D., Tkachuk, G. A., Shay, B. L., Vincent, N., Pear, J. J., \& Skrabek, R. Q. (2018). Randomized controlled trial of online acceptance and commitment therapy for fibromyalgia. The fournal of Pain, 19(7), 741-753. https://doi.org/10.1016/j.jpain.2018.02.004

Simons, L. E., Smith, A., Kaczynski, K., \& Basch, M. (2015). Living in fear of your child's pain: The parent fear of pain questionnaire. Pain, 156(4), 694-702. https://doi.org/10.1097/j.pain.000000000000100

Sunderland, M., Newby, J. M., \& Andrews, G. (2013). Health anxiety in Australia: Prevalence, comorbidity, disability and service use. The British fournal of Psychiatry, 202(1), 56-61. https://doi.org/10.1192/bjp.bp.111.103960

Thompson, M., \& Gauntlett-Gilbert, J. (2008). Mindfulness with children and adolescents: Effective clinical application. Clinical Child Psychology and Psychiatry, 13(3), 395-407. https://doi.org/10.1177/1359104508090603

Thomson, A. B., \& Page, L. A. (2007). Psychotherapies for hypochondriasis. Cochrane Database of Systematic Reviews, 2. https://doi.org/10.1002/14651858.CD006520.pub2 
Thorgaard, M. V., Frostholm, L., \& Rask, C. U. (2018). Childhood and family factors in the development of health anxiety: A systematic review. Children's Health Care, 47(2), 198-238. https://doi.org/10.1080/02739615.2017.1318390

Thorgaard, M. V., Frostholm, L., Walker, L., Jensen, J. S., Morina, B., Lindegaard, H., . . Rask, C. U. (2017). Health anxiety by proxy in women with severe health anxiety: A case control study. fournal of Anxiety Disorders, 52, 8-14. https://doi.org/10.1016/j.janxdis.2017.09.001

Trompetter, H. R., Bohlmeijer, E. T., Veehof, M. M., \& Schreurs, K. M. (2015). Internet-based guided self-help intervention for chronic pain based on acceptance and commitment therapy: A randomized controlled trial. fournal of Behavioral Medicine, 38(1), 66-80. https://doi.org/10.1007/s10865-014-9579-0

Van Dam, N. T., van Vugt, M. K., Vago, D. R., Schmalzl, L., Saron, C. D., Olendzki, A., . . Meyer, D. E. (2018). Mind the hype: A critical evaluation and prescriptive agenda for research on mindfulness and meditation. Perspectives on Psychological Science, 13(1), 36-61. https://doi.org/10.1177/1745691617709589

van Dessel, N., den Boeft, M., van der Wouden, J. C., Kleinstauber, M., Leone, S. S., Terluin, B., . . van Marwijk, H. (2014). Non-pharmacological interventions for somatoform disorders and medically unexplained physical symptoms (MUPS) in adults. Cochrane Database of Systematic Reviews, 11. https://doi.org/10.1002/14651858.CD011142

van Geelen, S. M., Rydelius, P. A., \& Hagquist, C. (2015). Somatic symptoms and psychological concerns in a general adolescent population: Exploring the relevance of DSM-5 somatic symptom disorder. Journal of Psychosomatic Research, 79(4), 251-258.

https://doi.org/10.1016/j.jpsychores.2015.07.012

van Ravesteijn, H., Lucassen, P., Bor, H., van Weel, C., \& Speckens, A. (2013). Mindfulness-based cognitive therapy for patients with medically unexplained symptoms: A randomized controlled trial. Psychotherapy and Psychosomatics, 82(5), 299-310. https://doi.org/10.1159/000348588

Veehof, M. M., Oskam, M. J., Schreurs, K. M., \& Bohlmeijer, E. T. (2011). Acceptance-based interventions for the treatment of chronic pain: A systematic review and meta-analysis. Pain, 152(3), 533-542. https://doi.org/10.1016/j.pain.2010.11.002

Veehof, M. M., Trompetter, H. R., Bohlmeijer, E. T., \& Schreurs, K. M. (2016). Acceptance- and mindfulness-based interventions for the treatment of chronic pain: A meta-analytic review. Cognitive Behaviour Therapy, 45(1), 5-31. https://doi.org/10.1080/16506073.2015.1098724

Visser, S., \& Bouman, T. K. (2001). The treatment of hypochondriasis: Exposure plus response prevention vs cognitive therapy. Behaviour Research and Therapy, 39(4), 423-442. https://doi.org/10.1016/S0005-7967(00)00022-X

Waelde, L. C., Feinstein, A. B., Bhandari, R., Griffin, A., Yoon, I. A., \& Golianu, B. (2017). A pilot study of mindfulness meditation for pediatric chronic pain. Children, 4(5), Article 32. https://doi.org/10.3390/children4050032

Wallace, D. P., Woodford, B., \& Connelly, M. (2016). Promoting psychological flexibility in parents of adolescents with chronic pain: Pilot study of an 8-week group intervention. Clinical Practice in Pediatric Psychology, 4(4), 405-416. https://doi.org/10.1037/cpp0000160 
Weck, F., Neng, J. M., Schwind, J., \& Hofling, V. (2015). Exposure therapy changes dysfunctional evaluations of somatic symptoms in patients with hypochondriasis (health anxiety): A randomized controlled trial. Fournal of Anxiety Disorders, 34, 1-7.

https://doi.org/10.1016/j.janxdis.2015.05.008

Wetherell, J. L., Afari, N., Rutledge, T., Sorrell, J. T., Stoddard, J. A., Petkus, A. J., . . Atkinson, J. H. (2011). A randomized, controlled trial of acceptance and commitment therapy and cognitivebehavioral therapy for chronic pain. Pain, 152(9), 2098-2107.

https://doi.org/10.1016/j.pain.2011.05.016

WHO. (1992). The ICD-10 classification of mental and behavioural disorders: Clinical descriptions and diagnostic guidelines. Geneva, Switzerland: World Health Organization.

Wicksell, R. K., Dahl, J., Magnusson, B., \& Olsson, G. L. (2005). Using acceptance and commitment therapy in the rehabilitation of an adolescent female with chronic pain: A case example. Cognitive and Behavioral Practice, 12(4), 415-423. https://doi.org/10.1016/S1077-7229(05)80069-0

Wicksell, R. K., Melin, L., Lekander, M., \& Olsson, G. L. (2009). Evaluating the effectiveness of exposure and acceptance strategies to improve functioning and quality of life in longstanding pediatric pain-A randomized controlled trial. Pain, 141(3), 248-257.

https://doi.org/10.1016/j.pain.2008.11.006

Wicksell, R. K., Melin, L., \& Olsson, G. L. (2007). Exposure and acceptance in the rehabilitation of adolescents with idiopathic chronic pain - A pilot study. European fournal of Pain, 11(3), 267-274. https://doi.org/10.1016/j.ejpain.2006.02.012

Williams, M. J., McManus, F., Muse, K., \& Williams, J. M. (2011). Mindfulness-based cognitive therapy for severe health anxiety (hypochondriasis): An interpretative phenomenological analysis of patients' experiences. British fournal of Clinical Psychology, 50(4), 379-397. https://doi.org/10.1111/j.2044-8260.2010.02000.x

Wright, K. D., \& Asmundson, G. J. (2003). Health anxiety in children: Development and psychometric properties of the childhood illness attitude scales. Cognitive Behaviour Therapy, 32(4), 194-202. https://doi.org/10.1080/16506070310014691

Zernicke, K. A., Campbell, T. S., Blustein, P. K., Fung, T. S., Johnson, J. A., Bacon, S. L., \& Carlson, L. E. (2013). Mindfulness-based stress reduction for the treatment of irritable bowel syndrome symptoms: A randomized wait-list controlled trial. International fournal of Behavioral Medicine, 20(3), 385-396. https://doi.org/10.1007/s12529-012-9241-6 